\title{
Dimension-six terms in the Standard Model Lagrangian ${ }^{1}$
}

\section{B. Grzadkowski, ${ }^{a}$ M. Iskrzyński, ${ }^{a}$ M. Misiak ${ }^{a, b}$ and J. Rosiek ${ }^{a}$}

${ }^{a}$ Institute of Theoretical Physics, University of Warsaw, Hoża 69, PL-00-681 Warsaw, Poland

${ }^{b}$ Institut für Theoretische Teilchenphysik, Karlsruhe Institute of Technology (KIT), D-76128 Karlsruhe, Germany

E-mail: Bohdan.Grzadkowski@fuw.edu.pl, mkisk@okwf.fuw.edu.pl, Mikolaj.Misiak@fuw.edu.pl, Janusz.Rosiek@fuw.edu.pl

ABSTRACT: When the Standard Model is considered as an effective low-energy theory, higher dimensional interaction terms appear in the Lagrangian. Dimension-six terms have been enumerated in the classical article by Buchmüller and Wyler [3]. Although redundance of some of those operators has been already noted in the literature, no updated complete list has been published to date. Here we perform their classification once again from the outset. Assuming baryon number conservation, we find $15+19+25=59$ independent operators (barring flavour structure and Hermitian conjugations), as compared to $16+35+29=80$ in ref. [3]. The three summed numbers refer to operators containing 0,2 and 4 fermion fields. If the assumption of baryon number conservation is relaxed, 5 new operators arise in the four-fermion sector.

Keywords: Standard Model, Beyond Standard Model

ARXIV EPRINT: $1008.4884 \mathrm{v} 2$

\footnotetext{
${ }^{1}$ This paper is based on the MSc thesis of the second author.
} 


\section{Contents}

1 Introduction 1

2 Notation and conventions 2

3 The complete set of dimension-five and -six operators 3

4 Comparison with ref. [3] 5

$\begin{array}{lll}5 & \text { Bosonic operator classification } & 7\end{array}$

6 Single-fermionic-current operator classification 10

$\begin{array}{lll}7 & \text { Four-fermion operator classification } & 13\end{array}$

8 Conclusions $\quad 15$

\section{Introduction}

The Standard Model (SM) of strong and electroweak interactions has been successfully tested to a great precision [1]. Nevertheless, it is commonly accepted that it constitutes merely an effective theory which is applicable up to energies not exceeding a certain scale $\Lambda$. A field theory valid above that scale should satisfy the following requirements:

(i) its gauge group should contain $\mathrm{SU}(3)_{C} \times \mathrm{SU}(2)_{L} \times \mathrm{U}(1)_{Y}$ of the $\mathrm{SM}$,

(ii) all the SM degrees of freedom should be incorporated either as fundamental or composite fields,

(iii) at low-energies, it should reduce to the SM, provided no undiscovered but weakly coupled light particles exist, like axions or sterile neutrinos.

In most of beyond-SM theories that have been considered to date, reduction to the SM at low energies proceeds via decoupling of heavy particles with masses of order $\Lambda$ or larger. Such a decoupling at the perturbative level is described by the Appelquist-Carazzone theorem [2]. This inevitably leads to appearance of higher-dimensional operators in the SM Lagrangian that are suppressed by powers of $\Lambda$

$$
\mathcal{L}_{\mathrm{SM}}=\mathcal{L}_{\mathrm{SM}}^{(4)}+\frac{1}{\Lambda} \sum_{k} C_{k}^{(5)} Q_{k}^{(5)}+\frac{1}{\Lambda^{2}} \sum_{k} C_{k}^{(6)} Q_{k}^{(6)}+\mathcal{O}\left(\frac{1}{\Lambda^{3}}\right),
$$




\begin{tabular}{|c|ccccc|c|}
\hline & \multicolumn{5}{|c|}{ fermions } & scalars \\
\hline field & $l_{L p}^{j}$ & $e_{R p}$ & $q_{L p}^{\alpha j}$ & $u_{R p}^{\alpha}$ & $d_{R p}^{\alpha}$ & $\varphi^{j}$ \\
\hline hypercharge $Y$ & $-\frac{1}{2}$ & -1 & $\frac{1}{6}$ & $\frac{2}{3}$ & $-\frac{1}{3}$ & $\frac{1}{2}$ \\
\hline
\end{tabular}

Table 1. The SM matter content

where $\mathcal{L}_{\mathrm{SM}}^{(4)}$ is the usual "renormalizable" part of the SM Lagrangian. It contains dimensiontwo and -four operators only. ${ }^{1}$ In the remaining terms, $Q_{k}^{(n)}$ denote dimension- $n$ operators, and $C_{k}^{(n)}$ stand for the corresponding dimensionless coupling constants (Wilson coefficients). Once the underlying high-energy theory is specified, all the coefficients $C_{k}^{(n)}$ can be determined by integrating out the heavy fields.

Our goal in this paper is to find a complete set of independent operators of dimension 5 and 6 that are built out of the SM fields and are consistent with the SM gauge symmetries. We do not rely on the original analysis of such operators by Buchmüller and Wyler [3] but rather perform the full classification once again from the outset. One of the reasons for repeating the analysis is the fact that many linear combinations of operators listed in ref. [3] vanish by the Equations Of Motion (EOMs). Such operators are redundant, i.e. they give no contribution to on-shell matrix elements, both in perturbation theory (to all orders) and beyond [4-9]. Although the presence of several EOM-vanishing combinations in ref. [3] has been already pointed out in the literature [10-13], no updated complete list has been published to date. Our final operator basis differs from ref. [3] also in the four-fermion sector where the EOMs play no role.

The article is organized as follows. Our notation and conventions are specified in section 2. The complete operator list is presented in section 3. Comparison with ref. [3] is outlined in section 4. Details of establishing operator bases in the zero-, two- and fourfermion sectors are described in sections 5,6 and 7, respectively. We conclude in section 8 .

\section{Notation and conventions}

The SM matter content is summarized in table 1 with isospin, colour, and generation indices denoted by $j=1,2, \quad \alpha=1,2,3$, and $p=1,2,3$, respectively. Chirality indices $(L, R)$ of the fermion fields will be skipped in what follows. Complex conjugate of the Higgs field will always occur either as $\varphi^{\dagger}$ or $\widetilde{\varphi}$, where $\widetilde{\varphi}^{j}=\varepsilon_{j k}\left(\varphi^{k}\right)^{\star}$, and $\varepsilon_{j k}$ is totally antisymmetric with $\varepsilon_{12}=+1$.

The well-known expression for $\mathcal{L}_{\mathrm{SM}}^{(4)}$ before Spontaneous Symmetry Breakdown (SSB) reads

$$
\begin{aligned}
\mathcal{L}_{\mathrm{SM}}^{(4)}= & -\frac{1}{4} G_{\mu \nu}^{A} G^{A \mu \nu}-\frac{1}{4} W_{\mu \nu}^{I} W^{I \mu \nu}-\frac{1}{4} B_{\mu \nu} B^{\mu \nu}+\left(D_{\mu} \varphi\right)^{\dagger}\left(D^{\mu} \varphi\right)+m^{2} \varphi^{\dagger} \varphi-\frac{1}{2} \lambda\left(\varphi^{\dagger} \varphi\right)^{2} \\
& +i(\bar{l} \not D l+\bar{e} \not D e+\bar{q} \not D q+\bar{u} \not D u+\bar{d} \not D d)-\left(\bar{l} \Gamma_{e} e \varphi+\bar{q} \Gamma_{u} u \widetilde{\varphi}+\bar{q} \Gamma_{d} d \varphi+\text { h.c. }\right),
\end{aligned}
$$

\footnotetext{
${ }^{1}$ Canonical dimensions of operators are determined from the field contents alone, excluding possible dimensionful coupling constants. The only dimension-two operator in $\mathcal{L}_{\mathrm{SM}}^{(4)}$ is $\varphi^{\dagger} \varphi$ in the Higgs mass term.
} 
where the Yukawa couplings $\Gamma_{e, u, d}$ are matrices in the generation space. We shall not consider SSB in this paper. Our sign convention for covariant derivatives is exemplified by

$$
\left(D_{\mu} q\right)^{\alpha j}=\left(\partial_{\mu}+i g_{s} T_{\alpha \beta}^{A} G_{\mu}^{A}+i g S_{j k}^{I} W_{\mu}^{I}+i g^{\prime} Y_{q} B_{\mu}\right) q^{\beta k}
$$

Here, $T^{A}=\frac{1}{2} \lambda^{A}$ and $S^{I}=\frac{1}{2} \tau^{I}$ are the $\mathrm{SU}(3)$ and $\mathrm{SU}(2)$ generators, while $\lambda^{A}$ and $\tau^{I}$ are the Gell-Mann and Pauli matrices, respectively. All the hypercharges $Y$ have been listed in table 1.

It is useful to define Hermitian derivative terms that contain $\varphi^{\dagger} \overleftarrow{D}_{\mu} \varphi \equiv\left(D_{\mu} \varphi\right)^{\dagger} \varphi$ as follows:

$$
\varphi^{\dagger} i \stackrel{\leftrightarrow}{D_{\mu}} \varphi \equiv i \varphi^{\dagger}\left(D_{\mu}-\overleftrightarrow{D_{\mu}}\right) \varphi \quad \text { and } \quad \varphi^{\dagger} i \stackrel{\leftrightarrow}{D_{\mu}^{I}} \varphi \equiv i \varphi^{\dagger}\left(\tau^{I} D_{\mu}-\overleftarrow{D_{\mu}} \tau^{I}\right) \varphi
$$

The gauge field strength tensors and their covariant derivatives read

$$
\begin{aligned}
G_{\mu \nu}^{A} & =\partial_{\mu} G_{\nu}^{A}-\partial_{\nu} G_{\mu}^{A}-g_{s} f^{A B C} G_{\mu}^{B} G_{\nu}^{C}, & & \left(D_{\rho} G_{\mu \nu}\right)^{A}=\partial_{\rho} G_{\mu \nu}^{A}-g_{s} f^{A B C} G_{\rho}^{B} G_{\mu \nu}^{C}, \\
W_{\mu \nu}^{I} & =\partial_{\mu} W_{\nu}^{I}-\partial_{\nu} W_{\mu}^{I}-g \varepsilon^{I J K} W_{\mu}^{J} W_{\nu}^{K}, & \left(D_{\rho} W_{\mu \nu}\right)^{I} & =\partial_{\rho} W_{\mu \nu}^{I}-g \varepsilon^{I J K} W_{\rho}^{J} W_{\mu \nu}^{K} \\
B_{\mu \nu} & =\partial_{\mu} B_{\nu}-\partial_{\nu} B_{\mu}, & D_{\rho} B_{\mu \nu} & =\partial_{\rho} B_{\mu \nu} .
\end{aligned}
$$

Dual tensors are defined by $\tilde{X}_{\mu \nu}=\frac{1}{2} \varepsilon_{\mu \nu \rho \sigma} X^{\rho \sigma} \quad\left(\varepsilon_{0123}=+1\right)$, where $X$ stands for $G^{A}$, $W^{I}$ or $B$.

The fermion kinetic terms in $\mathcal{L}_{\mathrm{SM}}^{(4)}$ are Hermitian up to total derivatives, i.e. $i \bar{\psi} \not D \psi$-h.c. $=\partial_{\mu}\left(\bar{\psi} \gamma^{\mu} \psi\right)$. Total derivatives of gauge-invariant objects in $\mathcal{L}_{\mathrm{SM}}$ are skipped throughout the paper, as they give no physical effects. At the dimension-five and -six levels, we encounter no gauge-invariant operators that are built out of non-abelian gauge fields only, and equal to total derivatives of gauge-variant objects. At the dimension-four level, the two possible such terms $\widetilde{G}_{\mu \nu}^{A} G^{A \mu \nu}=4 \varepsilon^{\mu \nu \rho \sigma} \partial_{\mu}\left(G_{\nu}^{A} \partial_{\rho} G_{\sigma}^{A}-\frac{1}{3} g_{s} f^{A B C} G_{\nu}^{A} G_{\rho}^{B} G_{\sigma}^{C}\right)$ and the analogous $\widetilde{W}_{\mu \nu}^{I} W^{I \mu \nu}$ should be understood as implicitly present on the r.h.s of eq. (2.1). They leave the Feynman rules and EOMs unaffected, showing up in topological quantum effects only [14-19].

\section{The complete set of dimension-five and -six operators}

This section is devoted to presenting our final results (derived in sections 5, 6 and 7) for the basis of independent operators $Q_{n}^{(5)}$ and $Q_{n}^{(6)}$. Their independence means that no linear combination of them and their Hermitian conjugates is EOM-vanishing up to total derivatives.

Imposing the SM gauge symmetry constraints on $Q_{n}^{(5)}$ leaves out just a single operator [20], up to Hermitian conjugation and flavour assignments. It reads

$$
Q_{\nu \nu}=\varepsilon_{j k} \varepsilon_{m n} \varphi^{j} \varphi^{m}\left(l_{p}^{k}\right)^{T} C l_{r}^{n} \equiv\left(\widetilde{\varphi}^{\dagger} l_{p}\right)^{T} C\left(\widetilde{\varphi}^{\dagger} l_{r}\right)
$$

where $C$ is the charge conjugation matrix. ${ }^{2} Q_{\nu \nu}$ violates the lepton number $L$. After the electroweak symmetry breaking, it generates neutrino masses and mixings. Neither $\mathcal{L}_{\mathrm{SM}}^{(4)}$

\footnotetext{
${ }^{2}$ In the Dirac representation $C=i \gamma^{2} \gamma^{0}$, with Bjorken and Drell [21] phase conventions.
} 


\begin{tabular}{|c|c|c|c|c|c|}
\hline \multicolumn{2}{|r|}{$X^{3}$} & \multicolumn{2}{|r|}{$\varphi^{6}$ and $\varphi^{4} D^{2}$} & \multicolumn{2}{|r|}{$\psi^{2} \varphi^{3}$} \\
\hline$Q_{G}$ & $f^{A B C} G_{\mu}^{A \nu} G_{\nu}^{B \rho} G_{\rho}^{C \mu}$ & $Q_{\varphi}$ & $\left(\varphi^{\dagger} \varphi\right)^{3}$ & $Q_{e \varphi}$ & $\left(\varphi^{\dagger} \varphi\right)\left(\bar{l}_{p} e_{r} \varphi\right)$ \\
\hline$Q_{\widetilde{G}}$ & $f^{A B C} \widetilde{G}_{\mu}^{A \nu} G_{\nu}^{B \rho} G_{\rho}^{C \mu}$ & $Q_{\varphi \square}$ & $\left(\varphi^{\dagger} \varphi\right) \square\left(\varphi^{\dagger} \varphi\right)$ & $Q_{u \varphi}$ & $\left(\varphi^{\dagger} \varphi\right)\left(\bar{q}_{p} u_{r} \widetilde{\varphi}\right)$ \\
\hline$Q_{W}$ & $\varepsilon^{I J K} W_{\mu}^{I \nu} W_{\nu}^{J \rho} W_{\rho}^{K \mu}$ & $Q_{\varphi D}$ & $\left(\varphi^{\dagger} D^{\mu} \varphi\right)^{\star}\left(\varphi^{\dagger} D_{\mu} \varphi\right)$ & $Q_{d \varphi}$ & $\left(\varphi^{\dagger} \varphi\right)\left(\bar{q}_{p} d_{r} \varphi\right)$ \\
\hline$Q_{\widetilde{W}}$ & $\varepsilon^{I J K} \widetilde{W}_{\mu}^{I \nu} W_{\nu}^{J \rho} W_{\rho}^{K \mu}$ & & & & \\
\hline \multicolumn{2}{|r|}{$X^{2} \varphi^{2}$} & \multicolumn{2}{|r|}{$\psi^{2} X \varphi$} & \multicolumn{2}{|r|}{$\psi^{2} \varphi^{2} D$} \\
\hline$Q_{\varphi G}$ & $\varphi^{\dagger} \varphi G_{\mu \nu}^{A} G^{A \mu \nu}$ & $Q_{e W}$ & $\left(\bar{l}_{p} \sigma^{\mu \nu} e_{r}\right) \tau^{I} \varphi W_{\mu \nu}^{I}$ & $Q_{\varphi l}^{(1)}$ & $\left(\varphi^{\dagger} i \stackrel{\leftrightarrow}{D_{\mu}} \varphi\right)\left(\bar{l}_{p} \gamma^{\mu} l_{r}\right)$ \\
\hline$Q_{\varphi \widetilde{G}}$ & $\varphi^{\dagger} \varphi \widetilde{G}_{\mu \nu}^{A} G^{A \mu \nu}$ & $Q_{e B}$ & $\left(\bar{l}_{p} \sigma^{\mu \nu} e_{r}\right) \varphi B_{\mu \nu}$ & $Q_{\varphi l}^{(3)}$ & $\left(\varphi^{\dagger} i \stackrel{\leftrightarrow}{D_{\mu}^{I}} \varphi\right)\left(\bar{l}_{p} \tau^{I} \gamma^{\mu} l_{r}\right)$ \\
\hline$Q_{\varphi W}$ & $\varphi^{\dagger} \varphi W_{\mu \nu}^{I} W^{I \mu \nu}$ & $Q_{u G}$ & $\left(\bar{q}_{p} \sigma^{\mu \nu} T^{A} u_{r}\right) \widetilde{\varphi} G_{\mu \nu}^{A}$ & $Q_{\varphi e}$ & $\left(\varphi^{\dagger} i \stackrel{\leftrightarrow}{D_{\mu}} \varphi\right)\left(\bar{e}_{p} \gamma^{\mu} e_{r}\right)$ \\
\hline$Q_{\varphi \widetilde{W}}$ & $\varphi^{\dagger} \varphi \widetilde{W}_{\mu \nu}^{I} W^{I \mu \nu}$ & $Q_{u W}$ & $\left(\bar{q}_{p} \sigma^{\mu \nu} u_{r}\right) \tau^{I} \widetilde{\varphi} W_{\mu \nu}^{I}$ & $Q_{\varphi q}^{(1)}$ & $\left(\varphi^{\dagger} i \stackrel{\leftrightarrow}{D_{\mu}} \varphi\right)\left(\bar{q}_{p} \gamma^{\mu} q_{r}\right)$ \\
\hline$Q_{\varphi B}$ & $\varphi^{\dagger} \varphi B_{\mu \nu} B^{\mu \nu}$ & $Q_{u B}$ & $\left(\bar{q}_{p} \sigma^{\mu \nu} u_{r}\right) \widetilde{\varphi} B_{\mu \nu}$ & $Q_{\varphi q}^{(3)}$ & $\left(\varphi^{\dagger} i \stackrel{\leftrightarrow}{D_{\mu}^{I}} \varphi\right)\left(\bar{q}_{p} \tau^{I} \gamma^{\mu} q_{r}\right)$ \\
\hline$Q_{\varphi \widetilde{B}}$ & $\varphi^{\dagger} \varphi \widetilde{B}_{\mu \nu} B^{\mu \nu}$ & $Q_{d G}$ & $\left(\bar{q}_{p} \sigma^{\mu \nu} T^{A} d_{r}\right) \varphi G_{\mu \nu}^{A}$ & $Q_{\varphi u}$ & $\left(\varphi^{\dagger} i \stackrel{\leftrightarrow}{D_{\mu}} \varphi\right)\left(\bar{u}_{p} \gamma^{\mu} u_{r}\right)$ \\
\hline$Q_{\varphi W B}$ & $\varphi^{\dagger} \tau^{I} \varphi W_{\mu \nu}^{I} B^{\mu \nu}$ & $Q_{d W}$ & $\left(\bar{q}_{p} \sigma^{\mu \nu} d_{r}\right) \tau^{I} \varphi W_{\mu \nu}^{I}$ & $Q_{\varphi d}$ & $\left(\varphi^{\dagger} i \stackrel{\leftrightarrow}{D_{\mu}} \varphi\right)\left(\bar{d}_{p} \gamma^{\mu} d_{r}\right)$ \\
\hline$Q_{\varphi \widetilde{W} B}$ & $\varphi^{\dagger} \tau^{I} \varphi \widetilde{W}_{\mu \nu}^{I} B^{\mu \nu}$ & $Q_{d B}$ & $\left(\bar{q}_{p} \sigma^{\mu \nu} d_{r}\right) \varphi B_{\mu \nu}$ & $Q_{\varphi u d}$ & $i\left(\widetilde{\varphi}^{\dagger} D_{\mu} \varphi\right)\left(\bar{u}_{p} \gamma^{\mu} d_{r}\right)$ \\
\hline
\end{tabular}

Table 2. Dimension-six operators other than the four-fermion ones.

nor the dimension-six terms can do the job. Thus, consistency of the SM (as defined by eq. (1.1) and table 1) with observations crucially depends on this dimension-five term.

All the independent dimension-six operators that are allowed by the SM gauge symmetries are listed in tables 2 and 3. Their names in the left column of each block should be supplemented with generation indices of the fermion fields whenever necessary, e.g., $Q_{l q}^{(1)} \rightarrow Q_{l q}^{(1) p r s t}$. Dirac indices are always contracted within the brackets, and not displayed. The same is true for the isospin and colour indices in the upper part of table 3. In the lower-left block of that table, colour indices are still contracted within the brackets, while the isospin ones are made explicit. Colour indices are displayed only for operators that violate the baryon number $B$ (lower-right block of table 3 ). All the other operators in tables 2 and 3 conserve both $B$ and $L$.

The bosonic operators (classes $X^{3}, X^{2} \varphi^{2}, \varphi^{6}$ and $\varphi^{4} D^{2}$ ) are all Hermitian. Those containing $\widetilde{X}_{\mu \nu}$ are CP-odd, while the remaining ones are CP-even. For the operators containing fermions, Hermitian conjugation is equivalent to transposition of generation indices in each of the fermionic currents in classes $(\bar{L} L)(\bar{L} L),(\bar{R} R)(\bar{R} R),(\bar{L} L)(\bar{R} R)$, and $\psi^{2} \varphi^{2} D^{2}$ (except for $Q_{\varphi u d}$ ). For the remaining operators with fermions, Hermitian conjugates are not listed explicitly.

If $\mathrm{CP}$ is defined in the weak eigenstate basis then $Q_{\overline{(+)}} Q^{\dagger}$ are CP-odd (-even) for all the fermionic operators. It follows that $\mathrm{CP}$-violation by any of those operators requires a 


\begin{tabular}{|c|c|c|c|c|c|}
\hline & $(\bar{L} L)(\bar{L} L)$ & & $(\bar{R} R)(\bar{R} R)$ & & $(\bar{L} L)(\bar{R} R)$ \\
\hline$Q_{l l}$ & $\left(\bar{l}_{p} \gamma_{\mu} l_{r}\right)\left(\bar{l}_{s} \gamma^{\mu} l_{t}\right)$ & $Q_{e e}$ & $\left(\bar{e}_{p} \gamma_{\mu} e_{r}\right)\left(\bar{e}_{s} \gamma^{\mu} e_{t}\right)$ & & $\left(\bar{l}_{p} \gamma_{\mu} l_{r}\right)\left(\bar{e}_{s} \gamma^{\mu} e_{t}\right)$ \\
\hline$Q_{q q}^{(1)}$ & $\left(\bar{q}_{p} \gamma_{\mu} q_{r}\right)\left(\bar{q}_{s} \gamma^{\mu} q_{t}\right)$ & $Q_{u u}$ & $\left(\bar{u}_{p} \gamma_{\mu} u_{r}\right)\left(\bar{u}_{s} \gamma^{\mu} u_{t}\right)$ & & $\left(\bar{l}_{p} \gamma_{\mu} l_{r}\right)\left(\bar{u}_{s} \gamma^{\mu} u_{t}\right)$ \\
\hline$Q_{q q}^{(3)}$ & $\left(\bar{q}_{p} \gamma_{\mu} \tau^{I} q_{r}\right)\left(\bar{q}_{s} \gamma^{\mu} \tau^{I} q_{t}\right)$ & $Q_{d d}$ & $\left(\bar{d}_{p} \gamma_{\mu} d_{r}\right)\left(\bar{d}_{s} \gamma^{\mu} d_{t}\right)$ & $Q_{l d}$ & $\left(\bar{l}_{p} \gamma_{\mu} l_{r}\right)\left(\bar{d}_{s} \gamma^{\mu} d_{t}\right)$ \\
\hline$Q_{l q}^{(1)}$ & $\left(\bar{l}_{p} \gamma_{\mu} l_{r}\right)\left(\bar{q}_{s} \gamma^{\mu} q_{t}\right)$ & $Q_{e u}$ & $\left(\bar{e}_{p} \gamma_{\mu} e_{r}\right)\left(\bar{u}_{s} \gamma^{\mu} u_{t}\right)$ & & $\left(\bar{q}_{p} \gamma_{\mu} q_{r}\right)\left(\bar{e}_{s} \gamma^{\mu} e_{t}\right)$ \\
\hline$Q_{l q}^{(3)}$ & $\left(\bar{l}_{p} \gamma_{\mu} \tau^{I} l_{r}\right)\left(\bar{q}_{s} \gamma^{\mu} \tau^{I} q_{t}\right)$ & $Q_{e d}$ & $\left(\bar{e}_{p} \gamma_{\mu} e_{r}\right)\left(\bar{d}_{s} \gamma^{\mu} d_{t}\right)$ & $Q_{q u}^{(1)}$ & $\left(\bar{q}_{p} \gamma_{\mu} q_{r}\right)\left(\bar{u}_{s} \gamma^{\mu} u_{t}\right)$ \\
\hline & & $Q_{u d}^{(1)}$ & $\left(\bar{u}_{p} \gamma_{\mu} u_{r}\right)\left(\bar{d}_{s} \gamma^{\mu} d_{t}\right)$ & $Q_{q u}^{(8)}$ & $\left(\bar{q}_{p} \gamma_{\mu} T^{A} q_{r}\right)\left(\bar{u}_{s} \gamma^{\mu} T^{A} u_{t}\right)$ \\
\hline & & $Q_{u d}^{(8)}$ & $\left(\bar{u}_{p} \gamma_{\mu} T^{A} u_{r}\right)\left(\bar{d}_{s} \gamma^{\mu} T^{A} d_{t}\right)$ & $\mid Q_{q d}^{(1)}$ & $\left(\bar{q}_{p} \gamma_{\mu} q_{r}\right)\left(\bar{d}_{s} \gamma^{\mu} d_{t}\right)$ \\
\hline & & & 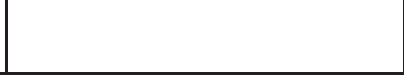 & $\mid Q_{q d}^{(8)}$ & $\left(\bar{q}_{p} \gamma_{\mu} T^{A} q_{r}\right)\left(\bar{d}_{s} \gamma^{\mu} T^{A} d_{t}\right)$ \\
\hline$(\bar{L} R)$ & $(\bar{R} L)$ and $(\bar{L} R)(\bar{L} R)$ & & $B$-vi & ting & \\
\hline$Q_{l e d q}$ & $\left(\bar{l}_{p}^{j} e_{r}\right)\left(\bar{d}_{s} q_{t}^{j}\right)$ & $Q_{d u q}$ & $\varepsilon^{\alpha \beta \gamma} \varepsilon_{j k}\left[\left(d_{p}^{\alpha}\right.\right.$ & $\left.C u_{r}^{\beta}\right]$ & {$\left[\left(q_{s}^{\gamma j}\right)^{T} C l_{t}^{k}\right]$} \\
\hline$Q_{q u q d}^{(1)}$ & $\left(\bar{q}_{p}^{j} u_{r}\right) \varepsilon_{j k}\left(\bar{q}_{s}^{k} d_{t}\right)$ & $Q_{q q u}$ & $\varepsilon^{\alpha \beta \gamma} \varepsilon_{j k}\left[\left(q_{p}^{\alpha}\right.\right.$ & $C q_{r}^{\beta k}$ & $k]\left[\left(u_{s}^{\gamma}\right)^{T} C e_{t}\right]$ \\
\hline$Q_{q u q d}^{(8)}$ & $\left(\bar{q}_{p}^{j} T^{A} u_{r}\right) \varepsilon_{j k}\left(\bar{q}_{s}^{k} T^{A} d_{t}\right)$ & $Q_{q q q}^{(1)}$ & $\varepsilon^{\alpha \beta \gamma} \varepsilon_{j k} \varepsilon_{m n}[(q$ & & $\left.{ }^{\beta k}\right]\left[\left(q_{s}^{\gamma m}\right)^{T} C l_{t}^{n}\right]$ \\
\hline$Q_{\text {lequ }}^{(1)}$ & $\left(\bar{l}_{p}^{j} e_{r}\right) \varepsilon_{j k}\left(\bar{q}_{s}^{k} u_{t}\right)$ & $Q_{q q q}^{(3)}$ & $\varepsilon^{\alpha \beta \gamma}\left(\tau^{I} \varepsilon\right)_{j k}\left(\tau^{I} \varepsilon\right)_{m n}$ & $\left(q_{p}^{\alpha j}\right)^{T}$ & $\left.{ }^{T} C q_{r}^{\beta k}\right]\left[\left(q_{s}^{\gamma m}\right)^{T} C l_{t}^{n}\right]$ \\
\hline$Q_{\text {lequ }}^{(3)}$ & $\left(\bar{l}_{p}^{j} \sigma_{\mu \nu} e_{r}\right) \varepsilon_{j k}\left(\bar{q}_{s}^{k} \sigma^{\mu \nu} u_{t}\right)$ & $Q_{d u u}$ & $\varepsilon^{\alpha \beta \gamma}\left[\left(d_{p}^{\alpha}\right)\right.$ & $u_{r}^{\beta}$ & {$\left[\left(u_{s}^{\gamma}\right)^{T} C e_{t}\right]$} \\
\hline
\end{tabular}

Table 3. Four-fermion operators.

non-vanishing imaginary part of the corresponding Wilson coefficient. However, one should remember that such a CP is not equivalent to the usual ("experimental") one defined in the mass eigenstate basis, just because the two bases are related by a complex unitary transformation.

Counting the entries in tables 2 and 3, we find 15 bosonic operators, 19 single-fermioniccurrent ones, and $25 B$-conserving four-fermion ones. In total, there are $15+19+25=59$ independent dimension-six operators, so long as $B$-conservation is imposed.

\section{Comparison with ref. [3]}

Comparing the $B$-conserving operators in tables 2 and 3 with eqs. (3.3)-(3.64) of ref. [3], one finds that

(i) The only operator missed in ref. [3] is $Q_{\text {lequ }}^{(3)}=\left(\bar{l}_{p}^{j} \sigma_{\mu \nu} e_{r}\right) \varepsilon_{j k}\left(\bar{q}_{s}^{k} \sigma^{\mu \nu} u_{t}\right)$. This fact has been already noticed in refs. $[22,23]$ where $\left(\bar{l}_{p}^{j} u_{t}^{\alpha}\right) \varepsilon_{j k}\left(\bar{q}_{s}^{k \alpha} e_{r}\right)=\frac{1}{8} Q_{l e q u}^{(3)}-\frac{1}{2} Q_{l e q u}^{(1)}$ was used instead. Phenomenological implications for top quark physics have been discussed, e.g., in ref. [24, 25]. 
(ii) One linear combination of the three $\varphi^{4} D^{2}$-class operators in eqs. (3.28) and (3.44) of ref. [3] must be redundant because this class contains two independent operators only. In fact, presence of all the three operators contradicts correct arguments given in section 3.5 of that paper.

(iii) The number of single-fermionic-current operators in ref. [3] becomes equal to ours after removing all the 16 operators with covariant derivatives acting on fermion fields (eqs. (3.30)-(3.37) and (3.57)-(3.59) there). As we shall show in section 6, all such operators are indeed redundant. This fact has been already discussed in refs. [10-12] for most of the cases. Note that removing those operators helps in eliminating multiple assignment of the same operator names in ref. [3].

(iv) Our use of $\stackrel{\leftrightarrow}{D_{\mu}}$ instead of $D_{\mu}$ in class $\psi^{2} \varphi^{2} D$ does not affect the formal operator counting, but actually reduces the number of terms to be considered. The point is that Hermitian conjugates of our operators with $\stackrel{\leftrightarrow}{D_{\mu}}$ have an identical form as the listed ones, so they do not need to be considered separately. On the other hand, using scalar field derivatives with a positive relative sign (opposite to that in eq. (2.3)) would give redundant operators only, i.e. linear combinations of the three $\psi^{2} \varphi^{3}$-class terms, EOM-vanishing objects, and total derivatives. This issue has been already noticed in ref. [13].

(v) Fierz identities (for anticommuting fermion fields) like the following one:

$$
\left(\bar{\psi}_{L} \gamma_{\mu} \psi_{L}\right)\left(\bar{\chi}_{L} \gamma^{\mu} \chi_{L}\right)=\left(\bar{\psi}_{L} \gamma_{\mu} \chi_{L}\right)\left(\bar{\chi}_{L} \gamma^{\mu} \psi_{L}\right)
$$

make 5 out of 29 four-fermion operators in ref. [3] linearly dependent on the others. For instance,

$$
\left(\bar{l}_{p} \gamma_{\mu} \tau^{I} l_{r}\right)\left(\bar{l}_{s} \tau^{I} \gamma^{\mu} l_{t}\right)=2\left(\bar{l}_{p}^{j} \gamma_{\mu} l_{r}^{k}\right)\left(\bar{l}_{s}^{k} \gamma^{\mu} l_{t}^{j}\right)-Q_{l l}^{p r s t}=2 Q_{l l}^{p t s r}-Q_{l l}^{p r s t}
$$

where the identity

$$
\tau_{j k}^{I} \tau_{m n}^{I}=2 \delta_{j n} \delta_{m k}-\delta_{j k} \delta_{m n}
$$

and eq. (4.1) have subsequently been used. Section 7 contains a full description of the four-fermion operator classification.

As far as the operator names and their normalization are concerned, our notation is close but not identical to the one of ref. [3]. Taking advantage of the need to modify the notation because of redundant operator removal, we do it in several places where convenience is the only issue.

The complete list of nomenclature and normalization changes reads:

(i) Unnecessary rationals are skipped in front of $Q_{\varphi G}, Q_{\varphi W}, Q_{\varphi B}, Q_{\varphi}, Q_{l l}, Q_{q q}^{(1)}, Q_{q q}^{(3)}$, $Q_{e e}, Q_{u u}$ and $Q_{d d}$.

(ii) $T^{A}$ instead of $\lambda^{A}$ are used in $Q_{u G}, Q_{d G}$ and $Q_{\ldots}^{(8)}$. 
(iii) Fierz transformation and multiplication by $(-2)$ is applied in our $(\bar{L} L)(\bar{R} R)$ class to avoid crossed colour and Dirac index contractions, and to make the notation somewhat more transparent. In addition, colour-Fierz transformations are applied to linear combinations of the last four operators of this class.

(iv) Operator names are changed in many cases to avoid multiple use of the same symbols, indicate the presence of essential fields, and make the nomenclature more systematic in the four-fermion sector. In particular, the names are modified for $Q_{\varphi W B}, Q_{\varphi \widetilde{W} B}$, $Q_{\varphi u d}$, as well as in the whole $(\bar{L} R)(\bar{R} L)$ and $(\bar{L} R)(\bar{L} R)$ classes.

One of the reasons for naming our operators with "Q" rather than with "O" is to indicate that many notational details have changed. As far as section 2 is concerned, we have followed ref. [3] everywhere except for sign conventions for the Yukawa couplings in eq. (2.1) and inside covariant derivatives (eq. (2.2)). The latter affects signs of operators in classes $X^{3}$ and $\psi^{2} X \varphi$.

\section{Bosonic operator classification}

Building blocks for the SM Lagrangian are the matter fields from table 1, the field strength tensors $X_{\mu \nu} \in\left\{G_{\mu \nu}^{A}, W_{\mu \nu}^{I}, B_{\mu \nu}\right\}$ and covariant derivatives of those objects. ${ }^{3}$ Using them and imposing just the global $\mathrm{SU}(3)_{C} \times \mathrm{SU}(2)_{L} \times \mathrm{U}(1)_{Y}$ symmetry is sufficient to find all the gauge-invariant operators in $\mathcal{L}_{\mathrm{SM}}$.

Purely bosonic operators must contain an even number of the Higgs fields $\varphi$ (because of the $\mathrm{SU}(2)_{L}$ representation tensor product constraints), and an even number of covariant derivatives $D$ (because all the Lorentz indices must be contracted). Since both $\varphi$ and $D$ have canonical dimension one, while $X$ has dimension two, no dimension-five operators can arise in the purely bosonic sector. The only possibilities for the dimension-six bosonic operator field contents are thus $X^{3}, X^{2} \varphi^{2}, X^{2} D^{2}, X \varphi^{4}, X D^{4}, X \varphi^{2} D^{2}, \varphi^{6}, \varphi^{4} D^{2}$ and $\varphi^{2} D^{4}$.

The class $X \varphi^{4}$ is empty because of the antisymmetry of $X$ and absence of any other objects with Lorentz indices to be contracted. We can also skip $X D^{4}$ because all the possible contractions (including those with $\varepsilon_{\mu \nu \rho \sigma}$ ) lead to appearance of at least one covariant derivative commutator $\left[D_{\mu}, D_{\nu}\right] \sim X_{\mu \nu}$, which moves us to the $X^{2} D^{2}$ class.

In the following, we shall show that all the possible operators in classes $\varphi^{2} D^{4}, \varphi^{2} X D^{2}$ and $X^{2} D^{2}$ reduce by the EOMs either to operators containing fermions or to classes $X^{3}$, $X^{2} \varphi^{2}, \varphi^{6}$ and $\varphi^{4} D^{2}$. Next, we shall verify that representatives of the latter four classes in table 2 indeed form a complete set of bosonic operators.

Since the necessary classical EOMs are going to be used at the $\mathcal{O}\left(\frac{1}{\Lambda^{2}}\right)$ level, and we are not interested in $\mathcal{O}\left(\frac{1}{\Lambda^{3}}\right)$ effects, we can neglect all the $\mathcal{O}\left(\frac{1}{\Lambda}\right)$ terms in the EOMs, i.e.

\footnotetext{
${ }^{3}$ If the requirement of gauge invariance was relaxed, gauge fields and their fully symmetrized derivatives like $\partial_{\left(\mu_{1}\right.} \ldots \partial_{\mu_{n}} G_{\nu)}^{A}$ would be the only additional objects. No expression depending on such terms could be gauge-invariant because one can simultaneously nullify all of them at any given spacetime point by an appropriate gauge transformation.
} 
derive them from $\mathcal{L}_{\mathrm{SM}}^{(4)}$ alone. We get then

$$
\begin{aligned}
\left(D^{\mu} D_{\mu} \varphi\right)^{j} & =m^{2} \varphi^{j}-\lambda\left(\varphi^{\dagger} \varphi\right) \varphi^{j}-\bar{e} \Gamma_{e}^{\dagger} l^{j}+\varepsilon_{j k} \bar{q}^{k} \Gamma_{u} u-\bar{d} \Gamma_{d}^{\dagger} q^{j}, \\
\left(D^{\rho} G_{\rho \mu}\right)^{A} & =g_{s}\left(\bar{q} \gamma_{\mu} T^{A} q+\bar{u} \gamma_{\mu} T^{A} u+\bar{d} \gamma_{\mu} T^{A} d\right), \\
\left(D^{\rho} W_{\rho \mu}\right)^{I} & =\frac{g}{2}\left(\varphi^{\dagger} i \stackrel{\leftrightarrow}{D_{\mu}^{I}} \varphi+\bar{l} \gamma_{\mu} \tau^{I} l+\bar{q} \gamma_{\mu} \tau^{I} q\right), \\
\partial^{\rho} B_{\rho \mu} & =g^{\prime} Y_{\varphi} \varphi^{\dagger} i \stackrel{\leftrightarrow}{D_{\mu}} \varphi+g^{\prime} \sum_{\psi \in\{l, e, q, u, d\}} Y_{\psi} \bar{\psi} \gamma_{\mu} \psi .
\end{aligned}
$$

Our ordering of operator classes is such that those containing fewer covariant derivatives are considered to be "lower". Throughout the paper, operators are going to be reduced from higher to lower classes. For classes containing equal numbers of derivatives, ordering is defined by the number of $X$ tensors, i.e. lower classes contain fewer $X$ tensors.

$\varphi^{2} D^{4}$ In this class, we can restrict our attention to operators where all the derivatives act on a single $\varphi$ field, because other possibilities are equivalent to them up to total derivatives. Contractions with $\varepsilon_{\mu \nu \rho \sigma}$ can be ignored because they lead to appearance of $\left[D_{\mu}, D_{\nu}\right] \sim$ $X_{\mu \nu}$, which moves us to lower classes containing $X$. For the same reason, ordering of the covariant derivatives acting on $\varphi$ can be chosen at will. We use this freedom to get $D^{\mu} D_{\mu} \varphi$ as a part of each of the considered operators. This moves us by the EOM to lower classes $\varphi^{4} D^{2}, \psi^{2} \varphi D^{2}$, and dimension-four operators multiplied by $m^{2}$.

$\varphi^{2} X D^{2}$ Here, we allow for $X$ being possibly dual, and forget about $\varepsilon_{\mu \nu \rho \sigma}$ otherwise. Indices of $X$ cannot be contracted with themselves, so they need to be contracted with both derivatives. We need to consider three cases: (i) Each of the derivatives acts on a different $\varphi$. We can eliminate this possibility "by parts", ignoring total derivatives. (ii) Both derivatives act on a single object. We obtain $\left[D_{\mu}, D_{\nu}\right] \sim X_{\mu \nu}$ and get moved to the $\varphi^{2} X^{2}$ class. (iii) One of the derivatives acts on $X$, and one on $\varphi$. We can take advantage either of the gauge field EOM (for the usual tensor) or of the Bianchi identity $D^{\rho} \widetilde{X}_{\rho \mu}=0$ (for the dual tensor). The EOM moves us to lower classes $\varphi^{4} D^{2}$ and $\psi^{2} \varphi^{2} D$.

$X^{2} D^{2}$ Similarly to the $\varphi^{2} D^{4}$ case, we can restrict our attention to operators where all the derivatives act on a single tensor. If both derivatives are contracted with $\varepsilon_{\mu \nu \rho \sigma}$ or with a single tensor, we obtain $\left[D_{\mu}, D_{\nu}\right] \sim X_{\mu \nu}$, and get moved to the $X^{3}$ class. Other contractions with $\varepsilon_{\mu \nu \rho \sigma}$ produce dual tensors. Thus, we allow the non-differentiated tensor to be possibly dual, and forget about $\varepsilon_{\mu \nu \rho \sigma}$ otherwise. If each of the derivatives is contracted with a different tensor, we can use $\left[D_{\mu}, D_{\nu}\right] \sim X_{\mu \nu}$ to choose their ordering in such a way that $D^{\rho} X_{\rho \mu}$ arises. In consequence, the operator gets reduced by the EOM to lower classes $\varphi^{2} X D^{2}$ and $\psi^{2} X D$.

The last possibility to consider is when the two derivatives are contracted with themselves:

$$
\stackrel{(\widetilde{X}}{ }^{\mu \nu} D^{\rho} D_{\rho} X_{\mu \nu}=-\stackrel{(\widetilde{X}}{ }^{\mu \nu}\left(D^{\rho} D_{\mu} X_{\nu \rho}+D^{\rho} D_{\nu} X_{\rho \mu}\right)=X^{3}+\varphi^{2} X D^{2}+\psi^{2} X D+E,
$$


In the first step, the Bianchi identity $D_{[\rho} X_{\mu \nu]}=0$ has been used. Next, $\left[D_{\rho}, D_{\alpha}\right] \sim X_{\rho \alpha}$ followed by the EOM for $X$ have been applied. The symbol $E$ stands for EOM-vanishing operators.

$X^{3}$ Here we begin to encounter classes whose representatives do appear in table 2. To indicate that the tensors may be different, we denote them by $X, Y$ and $Z$ in this paragraph. Allowing one of them to be dual, we can forget about $\varepsilon_{\mu \nu \rho \sigma}$ otherwise. The only non-vanishing and independent contraction of Lorentz indices reads $X_{\mu}{ }^{\nu} Y_{\nu}{ }^{\rho} Z_{\rho}^{\mu}$. This implies that all the three tensors must be different, because $X_{\alpha \mu} X_{\beta \nu} Z^{\mu \nu} g^{\alpha \beta}=0$ by the antisymmetry of $Z$. Moreover, neither of the two tensors can be related by duality because $X_{\mu}{ }^{\nu} \widetilde{X}_{\nu}{ }^{\rho}=-\frac{1}{4} \delta_{\mu}^{\rho} X_{\alpha \beta} \widetilde{X}^{\alpha \beta}$ is symmetric in the indices $(\mu \rho)$, while $Z$ is antisymmetric. It follows that (in particular) $B_{\mu}{ }^{\nu} W_{\nu}^{I \rho} \widetilde{W}_{\rho}^{I \mu}=0$, i.e. symmetric singlets in products of two adjoint representations are absent in the considered operator class. The only other option to get a gauge singlet from three different tensors is to use the structure constants $f A B C$ or $\varepsilon^{I J K}$. This leads us to a conclusion that the four $X^{3}$-class operators listed in table 2 are indeed the only possibilities.

$X^{2} \varphi^{2}$ The Higgs field products combine to singlets or triplets of $\mathrm{SU}(2)_{L}$. Hypercharge constraints imply that they must be of the form $\varphi^{\dagger} \varphi$ or $\varphi^{\dagger} \tau^{I} \varphi$ (but not, e.g., $\varphi^{\dagger} \tau^{I} \widetilde{\varphi}$ ). The eight $X^{2} \varphi^{2}$-class operators in table 2 contain all the possible contractions of two fieldstrength tensors that form singlets or triplets of $\mathrm{SU}(2)_{L}$, and singlets of $\mathrm{SU}(3)_{C}$.

$\varphi^{6}$ For the total hypercharge to vanish, exactly three of the Higgs fields must be complex conjugated. Grouping the six fields into $\varphi^{\star} \varphi$ pairs, and writing them as in the previous case, we are led to consider tensor products of singlets and triplets of $\mathrm{SU}(2)_{L}$. Three triplets can combine to an overall singlet only in a fully antisymmetric manner, which gives zero in our case because all the triplets are identical $\left(\varepsilon^{I J K}\left(\varphi^{\dagger} \tau^{I} \varphi\right)\left(\varphi^{\dagger} \tau^{J} \varphi\right)\left(\varphi^{\dagger} \tau^{K} \varphi\right)=0\right)$. Two triplets and one singlet combine to an overall singlet as $\left(\varphi^{\dagger} \tau^{I} \varphi\right)\left(\varphi^{\dagger} \tau^{I} \varphi\right)\left(\varphi^{\dagger} \varphi\right)$ that equals to $\left(\varphi^{\dagger} \varphi\right)^{3}$ thanks to eq. (4.3). Thus, the only independent operator in the considered class is the very $\left(\varphi^{\dagger} \varphi\right)^{3}$.

$\varphi^{4} D^{2}$ Hypercharge constraints imply that exactly two $\varphi$ fields must be complexconjugated. Since the two derivatives must be contracted, either they act on two different $\varphi$ fields, or the EOM moves the operator to lower classes. If they act on two conjugated or two unconjugated fields, we eliminate those possibilities "by parts". If one of them acts on a conjugated field, and the other on an unconjugated one, our $\mathrm{SU}(2)_{L}$ tensor product contains four distinct fundamental representations, which means that exactly two independent singlets must be present. Below, we write them on the l.h.s. as products of triplets and singlets, while the r.h.s. explains (via the Leibniz rule) what combinations give the two simple $\varphi^{4} D^{2}$-class operators in table 2 :

$$
\begin{aligned}
& \left(\varphi^{\dagger} \tau^{I} \varphi\right)\left[\left(D_{\mu} \varphi\right)^{\dagger} \tau^{I}\left(D^{\mu} \varphi\right)\right] \stackrel{(4.3)}{=} 2\left(\varphi^{\dagger} D^{\mu} \varphi\right)^{\star}\left(\varphi^{\dagger} D_{\mu} \varphi\right)-\left(\varphi^{\dagger} \varphi\right)\left[\left(D_{\mu} \varphi\right)^{\dagger}\left(D^{\mu} \varphi\right)\right], \\
& \left(\varphi^{\dagger} \varphi\right)\left[\left(D_{\mu} \varphi\right)^{\dagger}\left(D^{\mu} \varphi\right)\right] \stackrel{(5.1)}{=} \frac{1}{2}\left(\varphi^{\dagger} \varphi\right) \square\left(\varphi^{\dagger} \varphi\right)+\psi^{2} \varphi^{3}+\varphi^{6}+m^{2} \varphi^{4}+E .
\end{aligned}
$$




\section{Single-fermionic-current operator classification}

To make general arguments simple, it is convenient to think first in terms of only lefthanded fermions $\psi \in\left\{l, e^{c}, q, u^{c}, d^{c}\right\}$, i.e. to use charge conjugates of the $\mathrm{SU}(2)_{L}$-singlet fermions as fundamental fields. In such a case, we have only three possibilities for fermionic currents (up to h.c.): $\bar{\psi}_{1} \gamma_{\mu} \psi_{2}, \psi_{1}^{T} C \psi_{2}$ and $\psi_{1}^{T} C \sigma_{\mu \nu} \psi_{2}$. Considering bosonic objects with appropriate numbers of Lorentz indices and ignoring $X_{\mu}^{\mu}=0$, complete sets of building blocks for our operators are easily determined for each of the currents. They read ${ }^{4}$

$$
\begin{array}{rll}
\bar{\psi}_{1} \gamma_{\mu} \psi_{2}: & (\varphi D), & \left(X D, \varphi^{2} D, D^{3}\right), \\
\psi_{1}^{T} C \psi_{2}: & \left(\varphi^{2}, D^{2}\right), & \left(\varphi^{3}, \varphi D^{2}\right), \\
\psi_{1}^{T} C \sigma_{\mu \nu} \psi_{2}: & \left(X, D^{2}\right), & \left(X \varphi, \varphi D^{2}\right) .
\end{array}
$$

A brief look into table 1 ensures that hypercharges of the currents involving $C$ never vanish, while hypercharges of the vector currents never equal $\pm 1 / 2$. Consequently, classes $\psi^{2} X, \psi^{2} D^{2}$ and $\psi^{2} \varphi D$ are empty. Moreover, the Higgs field products in class $\psi^{2} \varphi^{2}$ must give non-zero hypercharges, in which case the only possibilities are \pm 1 . There is only a single fermionic current that can compensate such a hypercharge, namely the one built out of two lepton doublets. Thus, we obtain the field content of the operator in eq. (3.1). The isospin structure of that operator is the only available one given the antisymmetry of $\varepsilon_{j k}$ and the presence of just a single Higgs doublet in the SM. This completes our discussion of dimension-five operators.

In the dimension-six case, the number of Higgs fields associated with scalar and tensor fermionic currents is always odd. Consequently, those currents must form isospin doublets. In the standard notation with right-handed singlets, they read $\bar{\psi}_{1} \psi_{2}$ and $\bar{\psi}_{1} \sigma_{\mu \nu} \psi_{2}$. Similarly, vector currents can only form isospin singlets or triplets, as they combine with even numbers of the Higgs fields. Therefore, even if the isospin singlets are taken right-handed, no vector currents with $C$ enter into our considerations. We shall thus return to the standard notation in what follows.

Classical EOMs for the quarks and leptons that we are going to use below read

$$
i \not D l=\Gamma_{e} e \varphi, \quad i \not D e=\Gamma_{e}^{\dagger} \varphi^{\dagger} l, \quad i \not D q=\Gamma_{u} u \widetilde{\varphi}+\Gamma_{d} d \varphi, \quad i \not D u=\Gamma_{u}^{\dagger} \widetilde{\varphi}^{\dagger} q, \quad i \not D d=\Gamma_{d}^{\dagger} \varphi^{\dagger} q .
$$

Apart from them, two simple Dirac-algebra identities need to be recalled, namely

$$
\gamma_{\mu} \gamma_{\nu}=g_{\mu \nu}-i \sigma_{\mu \nu}, \quad \gamma_{\mu} \gamma_{\nu} \gamma_{\rho}=g_{\mu \nu} \gamma_{\rho}+g_{\nu \rho} \gamma_{\mu}-g_{\mu \rho} \gamma_{\nu}-i \varepsilon_{\mu \nu \rho \sigma} \gamma^{\sigma} \gamma_{5}
$$

Let us now discuss all the dimension-six classes one-by-one. ${ }^{5}$

$\psi^{2} D^{3}$ Three covariant derivatives are contracted here with a certain $\bar{\psi} \gamma_{\mu} \psi$ current. Similarly as in the previously discussed classes $\varphi^{2} D^{4}$ and $X^{2} D^{2}$, we can remove derivatives acting on $\bar{\psi}$ "by parts", and choose ordering of the derivatives acting on $\psi$ at will. Choosing the ordering as in $\bar{\psi} D_{\mu} D^{\mu} \not D \psi$, we get an operator that reduces by the EOMs to class $\psi^{2} \varphi D^{2}$.

\footnotetext{
${ }^{4}$ Bosonic terms leading to dimension-five and -six operators are collected in separate brackets.

${ }^{5}$ There are six of them. Note that both the scalar and tensor currents occur in the $\psi^{2} \varphi D^{2}$ case.
} 
$\psi^{2} \varphi D^{2} \quad$ As follows from eq. (6.1), this class involves scalar and tensor fermion currents only. We remove the derivatives acting on $\bar{\psi}$ "by parts", and take into account that $\bar{\psi} \sigma^{\mu \nu} \psi D_{\mu} D_{\nu} \varphi$ and $\varphi \bar{\psi} \sigma^{\mu \nu} D_{\mu} D_{\nu} \psi$ belong actually to class $\psi^{2} X \varphi$ because $\left[D_{\mu}, D_{\nu}\right] \sim X_{\mu \nu}$. The four remaining possibilities $\left[\bar{\psi} \psi D_{\mu} D^{\mu} \varphi, \varphi \bar{\psi} D_{\mu} D^{\mu} \psi, \quad\left(D_{\mu} \varphi\right) \bar{\psi} \sigma^{\mu \nu} D_{\nu} \psi\right.$ and $\left.\left(D^{\mu} \varphi\right) \bar{\psi} D_{\mu} \psi\right]$ are EOM-reduced to lower classes as follows:

$$
\begin{aligned}
& \bar{\psi} \psi D_{\mu} D^{\mu} \varphi \stackrel{(5.1)}{=} \psi^{4}+\psi^{2} \varphi^{3}+m^{2} \psi^{2} \varphi+E, \\
& \varphi \bar{\psi} D_{\mu} D^{\mu} \psi \stackrel{(6.3)}{=} \varphi \bar{\psi} \not D \not D \psi+\psi^{2} X \varphi \stackrel{(6.2)}{=} \psi^{2} X \varphi+\psi^{2} \varphi^{2} D+E \\
&\left(D_{\mu} \varphi\right) \bar{\psi} \sigma^{\mu \nu} D_{\nu} \psi=\frac{i}{2}\left(D_{\mu} \varphi\right) \bar{\psi}\left(\gamma^{\mu} \not D-\not D \gamma^{\mu}\right) \psi=i\left(D_{\mu} \varphi\right) \bar{\psi} \gamma^{\mu} \not D \psi-i\left(D^{\mu} \varphi\right) \bar{\psi} D_{\mu} \psi \\
& \stackrel{(6.2)}{=}-i\left(D^{\mu} \varphi\right) \bar{\psi} D_{\mu} \psi+\psi^{2} \varphi^{2} D+E, \\
& 2\left(D^{\mu} \varphi\right) \bar{\psi} D_{\mu} \psi=\left(D^{\mu} \varphi\right) \bar{\psi}\left(\gamma_{\mu} \not D+\not D \gamma_{\mu}\right) \psi \\
&=\left(D^{\mu} \varphi\right) \bar{\psi} \gamma_{\mu} \not D \psi-\bar{\psi} \overleftarrow{D D} \gamma_{\mu} \psi D^{\mu} \varphi-\bar{\psi} \gamma^{\nu} \gamma^{\mu} \psi D_{\nu} D_{\mu} \varphi+T \\
& \stackrel{(6.2)}{=} \psi^{2} \varphi^{2} D+\psi^{4}+\psi^{2} \varphi^{3}+m^{2} \psi^{2} \varphi+\psi^{2} X \varphi+E+T
\end{aligned}
$$

where $T$ stands for a total derivative. In the last step above, one should realize that

$$
\begin{aligned}
\bar{\psi} \gamma^{\nu} \gamma^{\mu} \psi D_{\nu} D_{\mu} \varphi & \stackrel{(6.3)}{=} \bar{\psi} \psi D_{\mu} D^{\mu} \varphi-i \bar{\psi} \sigma^{\nu \mu} \psi D_{\nu} D_{\mu} \varphi \\
& \stackrel{(5.1)}{=} \psi^{4}+\psi^{2} \varphi^{3}+m^{2} \psi^{2} \varphi+\psi^{2} X \varphi+E .
\end{aligned}
$$

$\psi^{2} X D$ As in several previous cases, we allow for $X$ being possibly dual, and forget about $\varepsilon_{\mu \nu \rho \sigma}$ otherwise. Since we deal here with $\bar{\psi} \gamma_{\mu} \psi$ currents only, the derivative must be contracted with $X$. If it acts on $X$, we obtain either the gauge field EOM (for the usual tensor) or the Bianchi identity $D^{\rho} \widetilde{X}_{\rho \mu}=0$ (for the dual tensor). The EOM moves us to lower classes $\psi^{2} \varphi^{2} D$ and $\psi^{4}$. Removing "by parts" terms with derivatives acting on $\bar{\psi}$, we find that the only expression still to be considered is $X^{\mu \nu} \bar{\psi} \gamma_{\mu} D_{\nu} \psi$. It gets reduced to lower classes as follows:

$$
\begin{aligned}
X^{\mu \nu} \bar{\psi} \gamma_{\mu} D_{\nu} \psi= & \frac{1}{2} X^{\mu \nu} \bar{\psi}\left(\gamma_{\mu} \gamma_{\nu} \not D+\gamma_{\mu} \not D \gamma_{\nu}\right) \psi=\frac{1}{2} X^{\mu \nu} \bar{\psi}\left(\gamma_{\mu} \gamma_{\nu} \not D-\not D \gamma_{\mu} \gamma_{\nu}\right) \psi+X^{\mu \nu} \bar{\psi} \gamma_{\nu} D_{\mu} \psi \\
\stackrel{(*)}{=} & \frac{1}{4} X^{\mu \nu} \bar{\psi}\left(\gamma_{\mu} \gamma_{\nu} \not D-\not D \gamma_{\mu} \gamma_{\nu}\right) \psi=\frac{1}{4} X^{\mu \nu} \bar{\psi} \gamma_{\mu} \gamma_{\nu} \not D \psi+\frac{1}{4} \bar{\psi} \overleftarrow{D D} \gamma_{\mu} \gamma_{\nu} \psi X^{\mu \nu} \\
& +\frac{1}{4} \bar{\psi} \gamma_{\rho} \gamma_{\mu} \gamma_{\nu} \psi D^{\rho} X^{\mu \nu}+T \stackrel{(6.2)}{=} \psi^{2} X \varphi+\psi^{2} \varphi^{2} D+\psi^{4}+E+T
\end{aligned}
$$

In the third step above (denoted by $(*)$ ), we have taken into account that the last term in the preceding expression is equal to our initial operator but with an opposite sign. In the last step, we have used the equality

$\bar{\psi} \gamma_{\rho} \gamma_{\mu} \gamma_{\nu} \psi D^{\rho} X^{\mu \nu} \stackrel{(6.3)}{=} 2 \bar{\psi} \gamma^{\mu} \psi D^{\rho} X_{\rho \mu}-i \varepsilon_{\rho \mu \nu \sigma} \bar{\psi} \gamma^{\sigma} \gamma_{5} \psi D^{\rho} X^{\mu \nu}=\psi^{2} \varphi^{2} D+\psi^{4}+\frac{E}{(6.7)}$. 
Both the gauge field EOM and the Bianchi identity are necessary in eq. (6.7), irrespectively of whether the initial $X$ is dual or not.

$\psi^{2} \varphi^{3}$ According to the arguments given above eq. (6.2), the fermion current must be an isospin doublet and colour singlet of the form $\bar{\psi}_{1} \psi_{2}$, i.e. one of those present in the Yukawa terms in eq. (2.1). The number of conjugated and unconjugated scalar fields in $\varphi^{3}$ is fixed for each of the fermionic currents by hypercharge constraints. Combining those scalar fields into an isospin doublet is unique because one of the two doublets in $\hat{2} \otimes \hat{2} \otimes \hat{2}$ vanishes in each of the cases due to $\varphi^{\dagger} \widetilde{\varphi}=\varepsilon_{j k}\left(\varphi^{j}\right)^{\star}\left(\varphi^{k}\right)^{\star}=0=\varepsilon_{j k} \varphi^{j} \varphi^{k}$. Consequently, the only possibilities for this class are the Yukawa terms multiplied by $\varphi^{\dagger} \varphi$, as in the upper-right block of table 2 .

$\psi^{2} X \varphi$ The antisymmetric tensor and the single Higgs field enforce the fermion current to be an isospin doublet of the form $\bar{\psi}_{1} \sigma^{\mu \nu} \psi_{2}$. Vanishing total hypercharge can be obtained only if the Higgs field combines with the currents in analogy to the standard Yukawa terms in eq. (2.1). Couplings with $B_{\mu \nu}$ in table 2 show this analogy most transparently. The tensors $W_{\mu \nu}^{I}$ and $G_{\mu \nu}^{A}$ need to be contracted with isospin triplets and colour octets, respectively, which can be formed just in a single way for each of the cases, as in table 2. Dualizing the $X$ tensor in any of the $\psi^{2} X \varphi$-class operators in that table would not give anything new because of the identities $\varepsilon_{\alpha \beta \mu \nu} \sigma^{\mu \nu}=2 i \sigma_{\alpha \beta} \gamma_{5}$ and $\gamma_{5} \psi_{L, R}=\mp \psi_{L, R}$.

$\psi^{2} \varphi^{2} D$ If the derivative acts on any of the fermion fields, its contraction with the $\bar{\psi} \gamma_{\mu} \psi$ current produces EOMs and moves us to the previously discussed lower class $\psi^{2} \varphi^{3}$. Thus, it is sufficient to consider derivatives acting on the scalars only. The Higgs fields can form isospin singlets or triplets, and are colour singlets. The fermion currents must follow the same selection rules, which allows precisely the currents listed in the $\psi^{2} \varphi^{2} D$-class block of table 2, up to Hermitian conjugation of the $\bar{u} \gamma_{\mu} d$ current. Hypercharge constraints determine the number of conjugated and unconjugated Higgs fields. We begin with removing "by parts" derivatives acting on one of the scalars, and forming isospin singlets or triplets from products of $\varphi_{1}$ and $D_{\mu} \varphi_{2}$, according to the structure of the corresponding fermion currents, which gives unique expressions in all the cases. This way we get operators differing from the ones in table 2 only by the presence of $D$ instead of the $\stackrel{\leftrightarrow}{D}$. However, we cannot terminate at this point because the operators without $\stackrel{\leftrightarrow}{D}$ are not Hermitian, and we still need to check whether their Hermitian conjugates are independent from them or not. Such a question does not arise for any other block of tables 2 and 3 because all the other operators are either manifestly Hermitian (up to flavour permutations in the upper part of table 3) or their Hermitian conjugates are manifestly independent (due to absence of hypercharge-conjugated fermion pairs). Such a manifest independence occurs also in the case of $Q_{\varphi u d}$ in the considered class, so we leave it with the usual derivative. ${ }^{6}$ In the remaining seven cases (which contain hypercharge-neutral currents), we form combinations

${ }^{6}$ Actually, $\widetilde{\varphi}^{\dagger}\left(D_{\mu}-\overleftarrow{D_{\mu}}\right) \varphi=2 \widetilde{\varphi}^{\dagger} D_{\mu} \varphi$ 
with $\stackrel{\leftrightarrow}{D}$ as in table 2 , and supplement them with symmetrized combinations of the form

$$
\left[\varphi^{\dagger}\left(D_{\mu}+\overleftarrow{D_{\mu}}\right) \varphi\right] \bar{\psi} \gamma^{\mu} \psi=\left[\partial_{\mu}\left(\varphi^{\dagger} \varphi\right)\right] \bar{\psi} \gamma^{\mu} \psi=\left(\varphi^{\dagger} \varphi\right) \bar{\psi}(\not D+\overleftarrow{D D}) \psi+T=\psi^{2} \varphi^{3}+E+T
$$

Thus, the symmetrized combinations give redundant operators and can be ignored. At this point, our classification of all the single-fermionic-current operators has been completed.

\section{Four-fermion operator classification}

Four fermion operators are the most numerous but very easy to classify. As in the beginning of the previous section, we think first in terms of only left-handed fermions $\psi \in\left\{l, e^{c}, q, u^{c}, d^{c}\right\}$. Lorentz-singlet products of the fermionic currents (6.1) and their Hermitian conjugates never give field contents like $\psi \psi \psi \bar{\psi}$ or $\psi \bar{\psi} \bar{\psi} \bar{\psi}$. For the remaining options, we search for zero-hypercharge products without paying attention to whether they can form isospin or colour singlets. There are several hundreds of cases to be tested, which is done in less than a second by a simple computer algebra code. Apart from trivial results giving products of two zero-hypercharge currents, only a handful of other possible field contents are found, namely

$$
\left(\bar{l} \bar{e}^{c} d^{c} q\right),\left(q u^{c} q d^{c}\right),\left(l e^{c} q u^{c}\right),(q q q l),\left(d^{c} u^{c} u^{c} e^{c}\right),\left(q q \bar{u}^{c} \bar{e}^{c}\right),\left(q l \bar{u}^{c} \bar{d}^{c}\right),
$$

and their Hermitian conjugates. Apparently, none of them can be eliminated using $\mathrm{SU}(2)_{L}$ or $\mathrm{SU}(3)_{C}$ constraints. The first three are $B$-conserving, while the remaining four are $B$-violating.

In the cases with two $\psi$ and two $\bar{\psi}$ fields in eq. (7.1), it is enough to consider only a single pairing of the four fields into two $\bar{\psi}_{L} \gamma_{\mu} \psi_{L}$ currents. ${ }^{7}$ As far as $\mathrm{SU}(2)_{L}$ is concerned, in each case there are two doublet and two singlet fields, which gives us only one overall singlet. Finally, there is only one $\mathrm{SU}(3)_{C}$ singlet in $\hat{\overline{3}} \otimes \hat{3}$ for the $B$-conserving operator, and one in $\hat{3} \otimes \hat{3} \otimes \hat{3}$ for the $B$-violating ones. Consequently, we get just a single operator for each of the three considered field contents. They are given by $Q_{l e d q}, Q_{d u q}$ and $Q_{q q u}$ in table 3 after passing to the standard notation with right-handed $\mathrm{SU}(2)_{L}$ singlets.

In the remaining cases in eq. (7.1), four left-handed $\psi$ fields occur. Once both the scalar and tensor currents from eq. (6.1) are taken into account, only a single pairing of the fields into currents needs to be considered. ${ }^{8}$ Alternatively, one can use the Fierz identity

$$
\left(\psi_{1 L}^{T} C \sigma_{\mu \nu} \psi_{2 L}\right)\left(\psi_{3 L}^{T} C \sigma^{\mu \nu} \psi_{4 L}\right)=4\left(\psi_{1 L}^{T} C \psi_{2 L}\right)\left(\psi_{3 L}^{T} C \psi_{4 L}\right)+8\left(\psi_{1 L}^{T} C \psi_{4 L}\right)\left(\psi_{3 L}^{T} C \psi_{2 L}\right)
$$

to get rid of the tensor currents. We choose the latter option everywhere except for the $\left(l e^{c} q u^{c}\right)$ field content $\left(Q_{l e q u}^{(1) \dagger}\right.$ and $\left.Q_{l e q u}^{(3) \dagger}\right)$, where we want to retain colour index contractions within the currents. In the three other cases $\left(\left(q u^{c} q d^{c}\right),(q q q l)\right.$ and $\left.\left(d^{c} u^{c} u^{c} e^{c}\right)\right)$, considering two different pairings amounts merely to a different generation index assignment, because two fields of the same type are always present. Once the fields are paired into currents, we

\footnotetext{
${ }^{7}$ There is only one $\mathrm{SL}(2, \mathbb{C})$ singlet in $\left(0, \frac{1}{2}\right) \otimes\left(0, \frac{1}{2}\right) \otimes\left(\frac{1}{2}, 0\right) \otimes\left(\frac{1}{2}, 0\right)$, which shows up in eq. (4.1).

${ }^{8}$ There are only two $\mathrm{SL}(2, \mathbb{C})$ singlets in $\left(\frac{1}{2}, 0\right) \otimes\left(\frac{1}{2}, 0\right) \otimes\left(\frac{1}{2}, 0\right) \otimes\left(\frac{1}{2}, 0\right)$.
} 
determine all the possible isospin and colour index contractions. Two possibilities exist in the $\left(q u^{c} q d^{c}\right)$ and $(q q q l)$ cases, while only a single one is present for $\left(d^{c} u^{c} u^{c} e^{c}\right)$. They are identified as $Q_{q u q d}^{(1) \dagger}, Q_{q u q d}^{(8) \dagger}, Q_{q q q}^{(1)}, Q_{q q q}^{(3)}$ and $Q_{d u u}^{\dagger}$.

This way we have completed establishing a basis for all the operators that cannot be written as products of zero-hypercharge currents, i.e. classes $(\bar{L} R)(\bar{R} L),(\bar{L} R)(\bar{L} R)$ and $B$-violating in table 3 . The $B$-violating ones are identical to those in ref. [26] where the original classification of refs. [20,27] was corrected. It is worth recalling that $Q_{q q q}^{(3)}$ vanishes in the flavour-diagonal case thanks to symmetry of all the three $\left(\tau^{I} \varepsilon\right)$ matrices, and to the equality $\psi_{1}^{T} C \psi_{2}=\psi_{2}^{T} C \psi_{1}$ that follows from the fermion field anticommutation and antisymmetry of the $C$ matrix.

If the field content of a four-fermion operator allows to write it as a product of two zero-hypercharge currents, we write it like that using the Fierz identity (4.1) if necessary. Next, we pass to the standard notation with right-handed $\mathrm{SU}(2)_{L}$ singlets, which splits the considered set into classes $(\bar{L} L)(\bar{L} L),(\bar{R} R)(\bar{R} R)$ and $(\bar{L} L)(\bar{R} R)$ in table 3 . It remains to convince oneself that the operators listed there indeed form complete bases for those classes. In the beginning, one should consider all the possible products of currents that form isospin singlets or triplets, and colour singlets or octets. Next, it is possible to eliminate several cases in the $(\bar{L} L)(\bar{L} L)$ and $(\bar{R} R)(\bar{R} R)$ classes using the relation (4.3) together with

$$
T_{\alpha \beta}^{A} T_{\kappa \lambda}^{A}=\frac{1}{2} \delta_{\alpha \lambda} \delta_{\kappa \beta}-\frac{1}{6} \delta_{\alpha \beta} \delta_{\kappa \lambda},
$$

and the Fierz identity (4.1) or its right-handed counterpart. It is essential to take into account that all the possible flavour assignments are included in table 3. One of such simplifications has been already shown in eq. (4.2). The remaining ones read

$$
\begin{aligned}
\left(\bar{u}_{p} \gamma_{\mu} T^{A} u_{r}\right)\left(\bar{u}_{s} T^{A} \gamma^{\mu} u_{t}\right) & \stackrel{(7.3)}{=} \frac{1}{2}\left(\bar{u}_{p}^{\alpha} \gamma_{\mu} u_{r}^{\beta}\right)\left(\bar{u}_{s}^{\beta} \gamma^{\mu} u_{t}^{\alpha}\right)-\frac{1}{6} Q_{u u}^{p r s t}=\frac{1}{2} Q_{u u}^{p t s r}-\frac{1}{6} Q_{u u}^{p r s t}, \quad \text { (7.4) } \\
\left(\bar{d}_{p} \gamma_{\mu} T^{A} d_{r}\right)\left(\bar{d}_{s} T^{A} \gamma^{\mu} d_{t}\right) & \stackrel{(7.3)}{=} \frac{1}{2}\left(\bar{d}_{p}^{\alpha} \gamma_{\mu} d_{r}^{\beta}\right)\left(\bar{d}_{s}^{\beta} \gamma^{\mu} d_{t}^{\alpha}\right)-\frac{1}{6} Q_{d d}^{p r s t}=\frac{1}{2} Q_{d d}^{p t s r}-\frac{1}{6} Q_{d d}^{p r s t}, \quad \text { (7.5) } \\
\left(\bar{q}_{p} \gamma_{\mu} T^{A} q_{r}\right)\left(\bar{q}_{s} T^{A} \gamma^{\mu} q_{t}\right) & \stackrel{(7.3)}{=} \frac{1}{2}\left(\bar{q}_{p}^{\alpha j} \gamma_{\mu} q_{r}^{\beta j}\right)\left(\bar{q}_{s}^{\beta k} \gamma^{\mu} q_{t}^{\alpha k}\right)-\frac{1}{6} Q_{q q}^{(1) p r s t} \\
& \stackrel{(4.1)}{=} \frac{1}{2}\left(\bar{q}_{p}^{\alpha j} \gamma_{\mu} q_{t}^{\alpha k}\right)\left(\bar{q}_{s}^{\beta k} \gamma^{\mu} q_{r}^{\beta j}\right)-\frac{1}{6} Q_{q q}^{(1) p r s t} \\
& \stackrel{(4.3)}{=} \frac{1}{4} Q_{q q}^{(3) p t s r}+\frac{1}{4} Q_{q q}^{(1) p t s r}-\frac{1}{6} Q_{q q}^{(1) p r s t}, \\
\left(\bar{q}_{p} \gamma_{\mu} T^{A} \tau^{I} q_{r}\right)\left(\bar{q}_{s} T^{A} \tau^{I} \gamma^{\mu} q_{t}\right) & \stackrel{(7.3)}{=} \frac{1}{2}\left(\bar{q}_{p}^{\alpha} \gamma_{\mu} \tau^{I} q_{r}^{\beta}\right)\left(\bar{q}_{s}^{\beta} \gamma^{\mu} \tau^{I} q_{t}^{\alpha}\right)-\frac{1}{6} Q_{q q}^{(3) p r s t} \\
& \stackrel{(4.3)}{=}\left(\bar{q}_{p}^{\alpha j} \gamma_{\mu} q_{r}^{\beta k}\right)\left(\bar{q}_{s}^{\beta k} \gamma^{\mu} q_{t}^{\alpha j}\right)-\frac{1}{2}\left(\bar{q}_{p}^{\alpha j} \gamma_{\mu} q_{r}^{\beta j}\right)\left(\bar{q}_{s}^{\beta k} \gamma^{\mu} q_{t}^{\alpha k}\right)-\frac{1}{6} Q_{q q}^{(3) p r s t} \\
& \stackrel{(4.1)}{=} Q_{q q}^{(1) p t s r}-\frac{1}{2}\left(\bar{q}_{p}^{\alpha j} \gamma_{\mu} q_{t}^{\alpha k}\right)\left(\bar{q}_{s}^{\beta k} \gamma^{\mu} q_{r}^{\beta j}\right)-\frac{1}{6} Q_{q q}^{(3) p r s t} \\
& \stackrel{(4.3)}{=}-\frac{1}{4} Q_{q q}^{(3) p t s r}+\frac{3}{4} Q_{q q}^{(1) p t s r}-\frac{1}{6} Q_{q q}^{(3) p r s t} .
\end{aligned}
$$

Establishing the above relations completes the proof that our four-fermion operator set in table 3 is indeed exhaustive. 


\section{Conclusions}

A tremendous simplification of the operator basis by the EOMs can be appreciated by comparing our table 2 that contains 34 entries with ref. [28] where 106 operators involving bosons are present because no EOM-reduction has been applied. Going down from 106 to 51 with the help of EOMs in ref. [3] has been a partial success. It is really amazing that no author of almost 600 papers that quoted ref. [3] over 24 years has ever decided to rederive the operator basis from the outset to check its correctness. As the current work shows, the exercise has been straightforward enough for an M. Sc. thesis [29, 30]. It has required no extra experience with respect to what was standard already in the 1980's.

From the phenomenological standpoint, it is hard to overestimate the importance of knowing the explicit form of power-suppressed terms in the SM Lagrangian. Although their overall number is sizeable, usually very few of them contribute to a given process. For instance, anomalous $W t b$ couplings that can be well tested at the LHC are described by four operators only $\left(Q_{u W}, Q_{d W}, Q_{\varphi q}^{(3)}\right.$ and $\left.Q_{\varphi u d}\right)[12,13,31]$. Given 14 operators in the dimension-four Lagrangian (2.1), it is actually quite surprising that no more than 59 operators arise at the dimension-six level.

It is interesting to note that if the underlying beyond-SM model is a weakly coupled (perturbative) gauge theory, operators containing field-strength tensors in table 2 cannot be tree-level generated [23]. In consequence, their Wilson coefficients $C_{k}$ are typically $\mathcal{O}\left(\frac{1}{16 \pi^{2}}\right)$. Thus, so long as we are interested in operators with $\mathcal{O}(1)$ coefficients only, as little as 14 entries of table 2 remain relevant. Investigations involving those operators can be found, e.g., in refs. [24, 25, 32].

Note added. While this article was being completed, a new paper [33] on four-fermion operator classification appeared on the arXiv. The number of independent $B$-conserving operators found there is the same as in our table 3. The key point are the identities (7.4)-(7.7) that have not remained unnoticed [34], but we are not aware of mentioning them in the literature previously in the context of correcting ref. [3].

\section{Acknowledgments}

We would like to thank Wilfried Buchmüller and Daniel Wyler for correspondence and discussions in years 2008-2010 concerning preparations to reanalyzing their operator classification [3]. We are grateful to Paweł Nurowski for helpful advice. The work of B.G. and M.M. has been supported in part by the Ministry of Science and Higher Education (Poland) as research project N N202 006334 (2008-11). B.G. and J.R. acknowledge support of the European Community within the Marie Curie Research \& Training Networks: "HEPTOOLS" (MRTN-CT-2006-035505), and "UniverseNet" (MRTN-CT-2006-035863). M.M. acknowledges support from the EU-RTN Programme "FLAVIAnet" (MRTN-CT-2006-035482) and from the DFG through the "Mercator" guest professorship programme. The work of J.R has been supported in part by the Ministry of Science and Higher Education (Poland) as research projects N N202 230337 (2009-12) and N N202 103838 (2010-12). 
Open Access. This article is distributed under the terms of the Creative Commons Attribution Noncommercial License which permits any noncommercial use, distribution, and reproduction in any medium, provided the original author(s) and source are credited.

\section{References}

[1] Particle Data Group, K. Nakamura et al., The Review of Particle Physics, J. Phys. G 37 (2010) 075021, http://pdg.lbl.gov.

[2] T. Appelquist and J. Carazzone, Infrared Singularities and Massive Fields, Phys. Rev. D 11 (1975) 2856 [SPIRES].

[3] W. Buchmüller and D. Wyler, Effective Lagrangian Analysis of New Interactions and Flavor Conservation, Nucl. Phys. B 268 (1986) 621 [SPIRES].

[4] H.D. Politzer, Power Corrections at Short Distances, Nucl. Phys. B 172 (1980) 349 [SPIRES].

[5] H. Kluberg-Stern and J.B. Zuber, Renormalization of Nonabelian Gauge Theories in a Background Field Gauge. 2. Gauge Invariant Operators, Phys. Rev. D 12 (1975) 3159 [SPIRES].

[6] C. Grosse-Knetter, Effective Lagrangians with higher derivatives and equations of motion, Phys. Rev. D 49 (1994) 6709 [hep-ph/9306321] [SPIRES].

[7] C. Arzt, Reduced effective Lagrangians, Phys. Lett. B 342 (1995) 189 [hep-ph/9304230] [SPIRES].

[8] H. Simma, Equations of motion for effective Lagrangians and penguins in rare $B$ decays, Z. Phys. C 61 (1994) 67 [hep-ph/9307274] [SPIRES].

[9] J. Wudka, Electroweak effective Lagrangians, Int. J. Mod. Phys. A 9 (1994) 2301 [hep-ph/9406205] [SPIRES].

[10] B. Grzadkowski, Z. Hioki, K. Ohkuma and J. Wudka, Probing anomalous top quark couplings induced by dimension-six operators at photon colliders, Nucl. Phys. B 689 (2004) 108 [hep-ph/0310159] [SPIRES].

[11] P.J. Fox, Z. Ligeti, M. Papucci, G. Perez and M.D. Schwartz, Deciphering top flavor violation at the LHC with B factories, Phys. Rev. D 78 (2008) 054008 [arXiv: 0704.1482] [SPIRES].

[12] J.A. Aguilar-Saavedra, A minimal set of top anomalous couplings, Nucl. Phys. B $\mathbf{8 1 2}$ (2009) 181 [arXiv:0811.3842] [SPIRES].

[13] J.A. Aguilar-Saavedra, A minimal set of top-Higgs anomalous couplings, Nucl. Phys. B 821 (2009) 215 [arXiv: 0904 . 2387] [SPIRES].

[14] A.A. Belavin, A.M. Polyakov, A.S. Schwartz and Y.S. Tyupkin, Pseudoparticle solutions of the Yang-Mills equations, Phys. Lett. B 59 (1975) 85 [SPIRES].

[15] G. 't Hooft, Computation of the quantum effects due to a four-dimensional pseudoparticle, Phys. Rev. D 14 (1976) 3432 [Erratum ibid. D 18 (1978) 2199] [SPIRES].

[16] R. Jackiw and C. Rebbi, Vacuum periodicity in a Yang-Mills quantum theory, Phys. Rev. Lett. 37 (1976) 172 [SPIRES].

[17] C.G. Callan Jr., R.F. Dashen and D.J. Gross, The structure of the gauge theory vacuum, Phys. Lett. B 63 (1976) 334 [SPIRES]. 
[18] V. Baluni, CP Violating Effects in QCD, Phys. Rev. D 19 (1979) 2227 [SPIRES].

[19] R.J. Crewther, P. Di Vecchia, G. Veneziano and E. Witten, Chiral Estimate of the Electric Dipole Moment of the Neutron in Quantum Chromodynamics, Phys. Lett. B 88 (1979) 123 [Erratum ibid. B 91 (1980) 487] [SPIRES].

[20] S. Weinberg, Baryon And Lepton Nonconserving Processes, Phys. Rev. Lett. 43 (1979) 1566 [SPIRES].

[21] J.D. Bjorken and S.D. Drell, Relativistic Quantum Mechanics, McGraw-Hill Inc., New York U.S.A. (1964).

[22] W. Buchmüller, B. Lampe and N. Vlachos, Contact Interactions and the Callan-Gross Relation, Phys. Lett. B 197 (1987) 379 [SPIRES].

[23] C. Arzt, M.B. Einhorn and J. Wudka, Patterns of deviation from the standard model, Nucl. Phys. B 433 (1995) 41 [hep-ph/9405214] [SPIRES].

[24] B. Grzadkowski, Z. Hioki and M. Szafranski, Four-Fermi effective operators in top-quark production and decay, Phys. Rev. D 58 (1998) 035002 [hep-ph/9712357] [SPIRES].

[25] B. Grzadkowski, Four Fermi effective operators at $e^{+} e^{-} \rightarrow \bar{t} t$, Acta Phys. Polon. B 27 (1996) 921 [hep-ph/9511279] [SPIRES].

[26] L.F. Abbott and M.B. Wise, The Effectiv Hamiltonian for Nucleon Decay, Phys. Rev. D 22 (1980) 2208 [SPIRES].

[27] F. Wilczek and A. Zee, Operator Analysis of Nucleon Decay, Phys. Rev. Lett. 43 (1979) 1571 [SPIRES].

[28] C.N. Leung, S.T. Love and S. Rao, Low-Energy Manifestations of a New Interaction Scale: Operator Analysis, Z. Phys. C 31 (1986) 433 [SPIRES].

[29] M. Iskrzyński, Classification of higher-dimensional operators in the Standard Model (in Polish), MSc Thesis, University of Warsaw, 2010.

[30] M. Iskrzyński, Corrected classification of higher-dimensional operators in the Standard Model, talk presented at the 17th IMPRS Workshop, Munich Germany (2010), http://indico.mppmu.mpg.de/indico/conferenceDisplay.py?confId $=901$.

[31] B. Grzadkowski and M. Misiak, Anomalous Wtb coupling effects in the weak radiative Bmeson decay, Phys. Rev. D 78 (2008) 077501 [arXiv:0802.1413] [SPIRES].

[32] B. Grzadkowski and J. Wudka, Higgs boson production at $e^{+} e^{-}$colliders: A model independent approach, Phys. Lett. B 364 (1995) 49 [hep-ph/9502415] [SPIRES].

[33] J.A. Aguilar-Saavedra, Effective four-fermion operators in top physics: a roadmap, arXiv: 1008.3562 [SPIRES].

[34] Z. Hioki, private communication. 